\title{
Basic demonstrations in diffractive and Fourier optics: use your eye as a Fourier transformer
}

\section{Erik Stijns}

Erik W. Stijns, "Basic demonstrations in diffractive and Fourier optics: use your eye as a Fourier transformer," Proc. SPIE 3190, Fifth International Topical Meeting on Education and Training in Optics, (8 December 1997); doi: $10.1117 / 12.294410$

SPIE Event: Fifth International Topical Meeting on Education and Training in Optics, 1997, Delft, Netherlands 
Basic demonstrations in diffractive and Fourier optics :

use your eye as a Fourier transformer

\title{
Erik Stijns
}

Vrije Universiteit Brussel, ALNA-TW, Pleinlaan 2,

B-1050 Brussel, Belgium

e-mail : estijns@vub.ac.be

\begin{abstract}
In this paper some setups are described for demonstrating the basics of diffractive and/or Fourier optics. It is first shown that the eye in fact can be used as a Fourier transformer : this makes the setups extremely simple.

When using one extra lens, a 4-f processor can be build, by which such properties as e.g. filtering can be demonstrated.

Keywords : Demonstrations, class-room demonstrations, diffractive optics, Fourier optics, eye.

\section{BASIC SETUP FOR FOURIER TRANSFORMATIONS}

Figure 1 shows the basic setup for making a Fourier transform(=F.T.) with a lens. An objectfield $u_{0}\left(x_{0}, y_{0}\right)$ in an objectplane $\Pi_{0}$ is transformed by a lens $L$ with focal length $f$. The image plane $\Pi_{i}$ is positioned at focal distance $f$ from
\end{abstract}

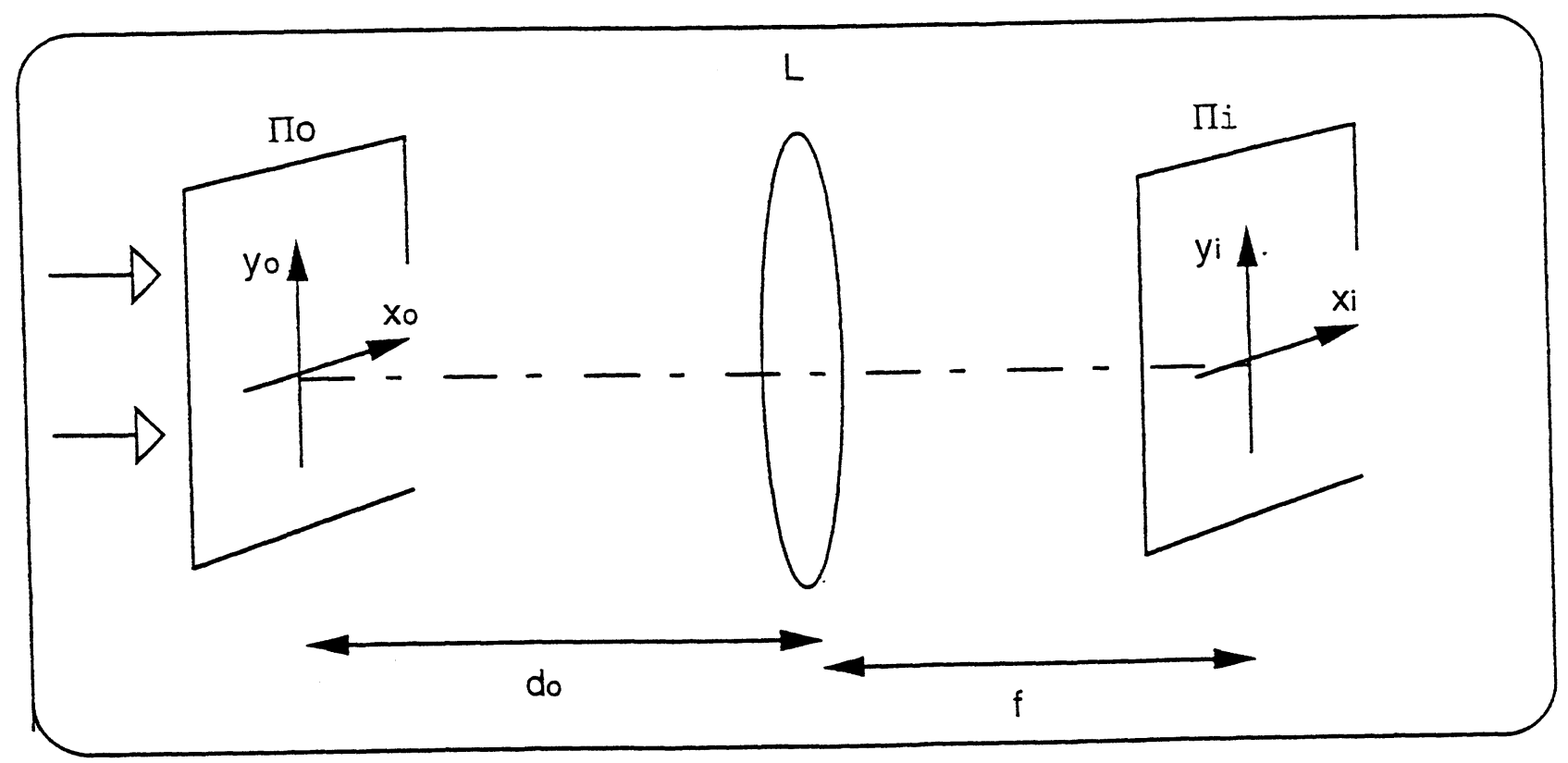

Figure 1 : Basic setup for Fouriertransforming 
that lens. The field in plane $\Pi_{\mathrm{i}}$ is given by ${ }^{1}$

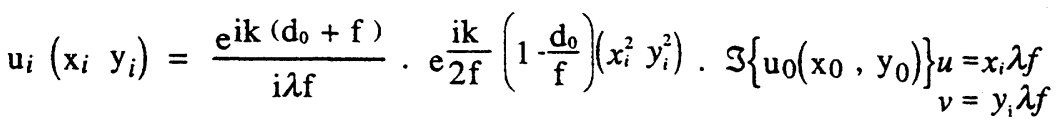

where $\mathfrak{3}\{\ldots\}$ stands for the Fourier transform and the subscripts transform the spatial frequencies $u$ and $v$ into the $x_{i}$ and $y_{i}$ coordinates of the image plane. When also the object plane is at focal distance $\left(\mathrm{d}_{0}=\mathrm{f}\right)$, then there exists an exact F.T. relationship between the two fields (up to a constant phase factor). In all other cases the F.T. is multiplied by a curved phase factor. However, when the image is observed on a screen, only the irradiance (often erronously called the "intensity") is observed, and this is independant of the phase. Consequently the image on a screen at focal distance is independant of the position of the object.

In these experiments it is always supposed that the light beam is coherent. This is realized in practical setups by illuminating a transparancy (in plane $\Pi_{0}$ ) with a collimated monochromatic and coherent light beam from a laser.

\section{THE EYE AS A FOURIER TRANSFORMER}

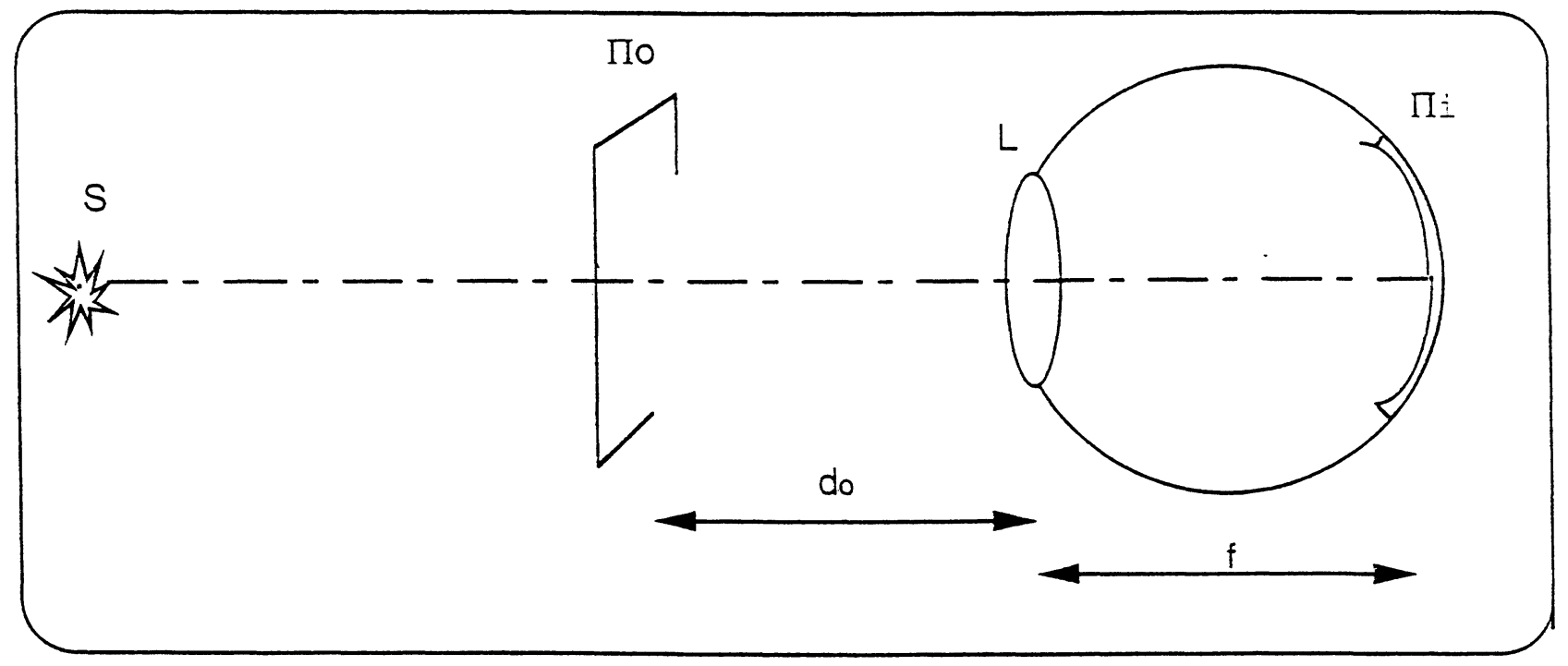

Figure 2 : the eye as a Fourier transformer 
Figure 2 shows an eye looking at a distant point $S$. When that point is at great distance, the eye is "relaxed" and the retina is at focal distance f from the lens $L$ (which is, for a "standard" eye, about $17 \mathrm{~mm}$ ) ${ }^{2}$. Suppose now that the point $\mathrm{S}$ is a light source, and put a transparancy $\Pi_{0}$ somewhere between $S$ and $L$. Then the image on the retina $\Pi_{i}$ is the Fourier transform of $\Pi_{0}$, on condition that the light is collimated and coherent. This in fact is not a real problem. Indeed : because the lens $\mathrm{L}$ and all planes considered are transverse to the optic axis, the longitudinal coherence is not relevant. The transverse coherence on the other hand depends on the transverse dimensions of the source; the smaller the latter, the better the transverse coherence. In our experiments we used a small ( $=$ "point") source, and never had problems with its coherence. The only practical problem comes from the colors. Indeed : if one looks at a polychromatic light source, then - because diffraction is proportional to wavelength - one obtains a multi-color image. This is maybe beautiful to observe, but surely difficult to interprete.

\section{EXPERIMENTS ON FOURIER TRANSFORMATIONS}

The experiment of Figure 2 is easy to perform. Any point source will do. We prefer to shine with a laser beam on a piece of white paper a few meters away and to look towards that lightpoint. This gives a bright point source, so that the experiments can be performed with normal ambient (classroom) illumination. Moreover the monochromaticity of the source simplifies the image on the retina and its interpretaion. What dimensions in objectplane $\Pi_{0}$ are necessary ? The resolving power of an eye is about 1 minute of arc, which is 0,3 millirad $^{2}$. This gives on the retina a distance between two spots of about $5 \mu \mathrm{m}$. A grating of spatial frequency $u$ in objectplane $\Pi_{0}$ gives spots in the imageplane at distances of

$$
\delta=u . \lambda . f
$$

So for obtaining $\delta>5 \mu \mathrm{m}$, u should be greater than about $500 \mathrm{~m}^{-1}$ when using $\lambda=500$ $\mathrm{nm}$. This means that the details of the object should be (much) smaller than about $2 \mathrm{~mm}$. This can easily be produced. Indeed we simply drew figures of about $1 \mathrm{~cm}$ on a paper and we took a picture of them on a black and white negative film, in which proces the linear magnification was almost $1 / 10$. Those negatives are directly used as object transparancies. In order to increase the light throughput we multiplicated the figure. It is known, indeed, that a shift of the object changes its F.T. only with a phase factor, leaving the irradiance pattern unaltered. Consequently, if we consider an array of identical objects in plane $\Pi_{0}$ instead of one single object, we obtain the same F.T. pattern, but with a higher irradiance. An example : for illustrating the F.T. of a letter A, we printed a square of identical letters A, large enough to fill the iris of the eye, as shown in figure 3 . 


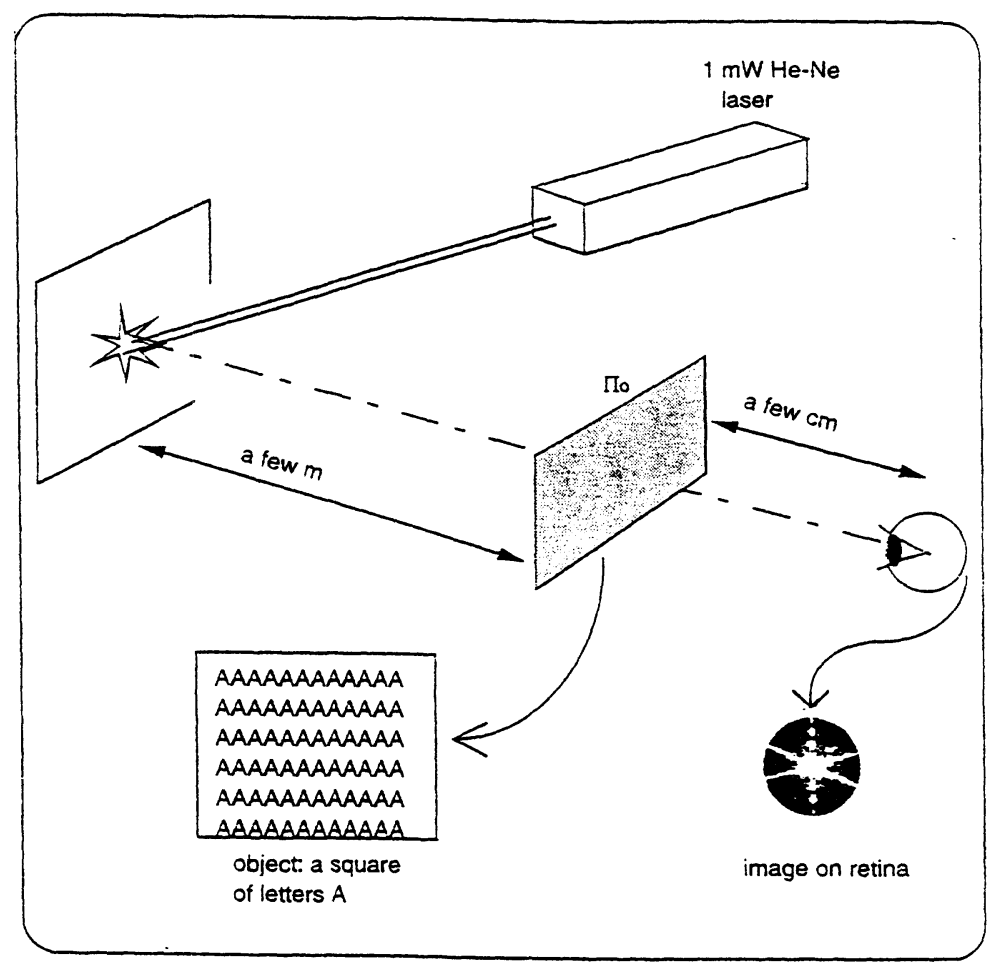

Figure 3 : use your eye as a Fourier transformer.

One observes that a movement of the objectfilm $\Pi_{0}$ longitudinally does not change the image on the retina, as should be expected from the explanation at the end of section 1. This also illustrates the fact that the image on the retina $\Pi_{i}$ is not the geometrical image of the object in $\Pi_{0}$. This observation also allows for a calibration of the Fourier plane. Indeed, when looking towards the point source $S$ on the wall, the spatial spectrum of the object is superimposed, in the retina of the eye, on the image of the wall. Consequently it is possible to use that wall for drawing the spatial frequency axis $u$ and $v$. Their calibration goes as follows (figure 4) 
Figure 4 : calibration of the spatial frequencies.

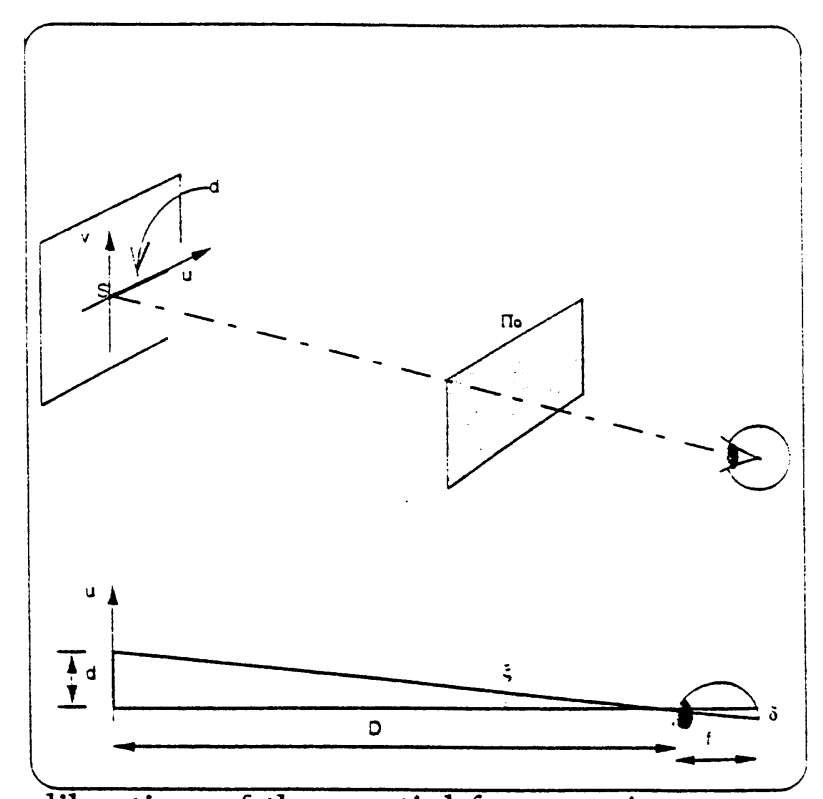

The angle $\xi$ equals (in the paraxial approximation)

$\xi=\frac{\delta}{f}$

which is, because of (2), equal to

$\xi=\mathrm{u} \delta$

On the other hand, $\xi$ also equals

$\xi=\frac{d}{D}$

and consequently

$\mathrm{d}=\mathrm{u} \lambda \mathrm{D}$

For example, when $\lambda=633 \mathrm{~nm}$ and $\mathrm{D}=5 \mathrm{~m}$, then a spatial frequency $\mathrm{u}$ of 11.p.mm. corresponds with a distance $\mathrm{d}$ of $3.2 \mathrm{~mm}$ on the wall.

These properties of the eye are not new, of course : it is already known for a long time that the eye produces, on its retina, a Fraunhofer diffraction pattern. Consequently, the setup of figure 2 or 3 has been used in the past for visualizing diffraction patterns $\mathrm{s}^{3,4}$. But, although a Fraunhofer diffraction pattern is of course nothing else than a scaled Fourier transform, the above mentioned experiments were in the past not explained as Fourier transforms. And, above all, it was apparantly not realized that other properties of F.T. can also be demonstrated with similar simple setups. This is the subject of next paragraphs. 


\section{4-f PROCESSOR}

The basic setup for optical processing is the so-called "4-f processor", which is shown in figure 5.

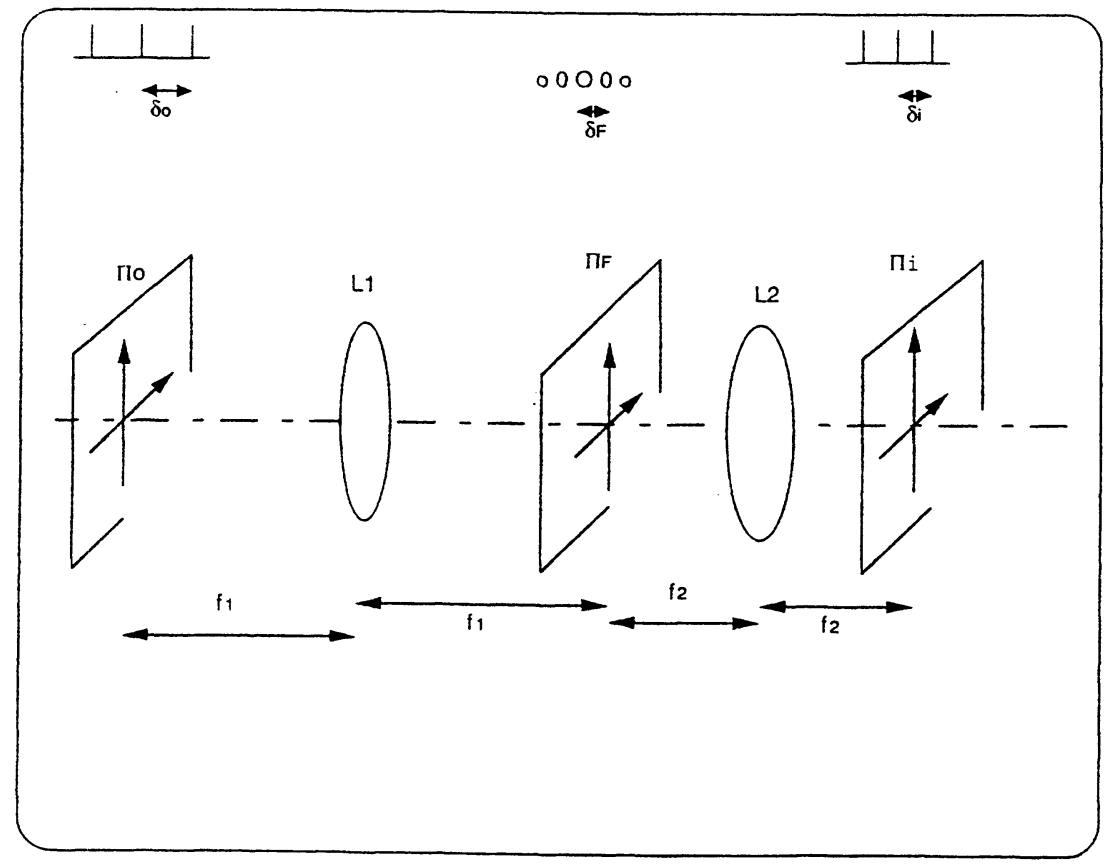

The object plane $\Pi_{0}$ is at focal distance $f_{1}$ from a first lens $L 1$, which produces the Fourier transform in its second focal plane $\Pi_{F}$. This in turn is again Fourier transformed by a second lens $L 2$, with focal length $f_{2}$. Finally one obtains, in image plane $\Pi_{i}$, an image of the object with a linear magnification of $M=-f_{2} / f_{1}$.

That image can be modified or "processed" by puting a filter somewhere between $\Pi_{0}$ and $\Pi_{\mathrm{i}}$; for simplifying the calculations and the design, that filter is usually positioned in the Fourier plane $\Pi_{\mathrm{F}}$.

\section{Calibration}

What feature sizes are necessary in order to obtain reasonable results ? A grating of spatial period $\delta_{0}$ in plane $\Pi_{0}$ gives spots in plane $\Pi_{F}$ at distances of

$$
\delta_{F}=\frac{\lambda_{\mathrm{f}_{1}}}{\delta_{0}}
$$

This gives a grating in the image plane $\Pi_{i}$ with spatial period

$$
\delta_{\mathrm{i}}=\frac{\mathrm{f}_{2}}{\mathrm{f}_{1}} \delta_{0}=\frac{\lambda_{\mathrm{f}}}{\delta_{\mathrm{F}}}
$$


For the second lens L2 in our experiments we will use of course the eye lens; so $f_{2}=$ $17 \mathrm{~mm}$ and $\delta_{\mathrm{i}}>5 \mu \mathrm{m}$ (as in section 3). This implies, when taking $\lambda=500 \mathrm{~nm}$, that

$\delta_{\mathrm{F}} \leq 1.7 \mathrm{~mm}$

Using for instance a lens $\mathrm{L} 1$ with $\mathrm{f}_{1}=10 \mathrm{~cm}$ implies that :

$\delta_{0} \geq \frac{\lambda_{\mathrm{f}_{1}}}{\mathrm{~d}_{\mathrm{F}}}=30 \mu m$

which corresponds with a spatial frequency smaller than about 30 1.p.mm.

\section{DEMONSTRATION OF THE ABBE-PORTER EXPERIMENT}

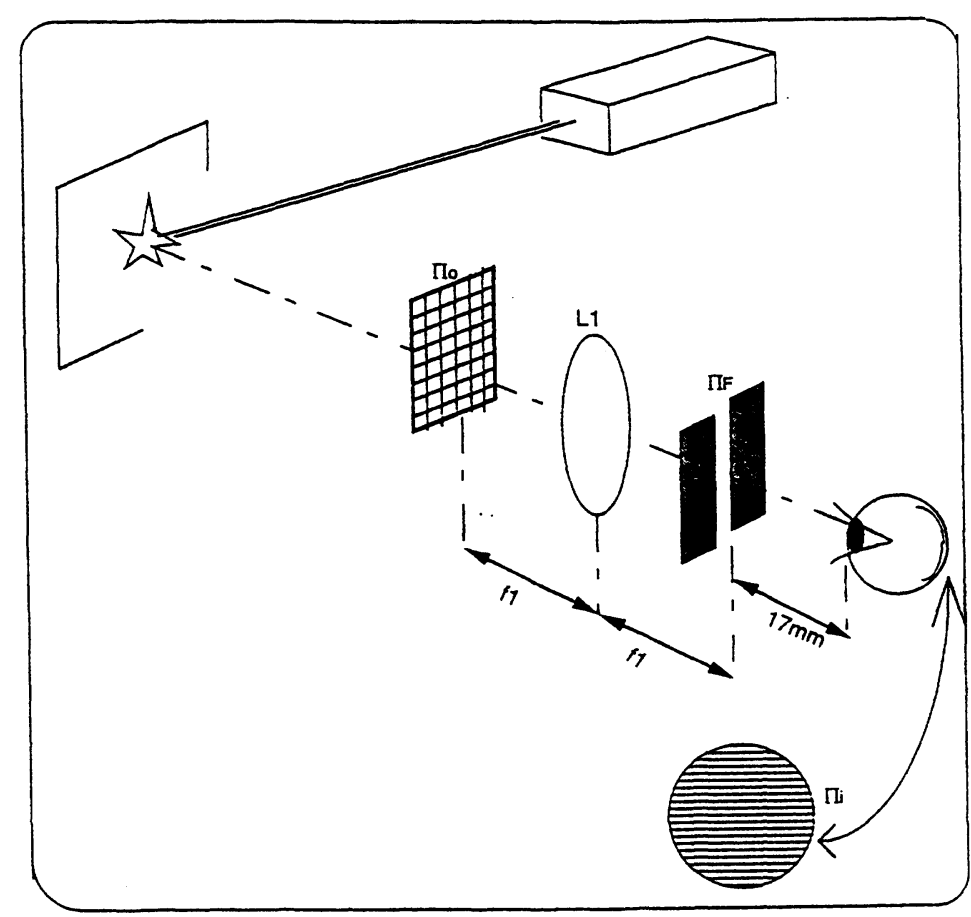

Figure $6:$ the Abbe-Porter experiment

Figure 6 shows how the theoretical setup of figure 5 can be transformed into an easyto-realize practical setup for illustrating the simpliest filtering setup, i.e. the experiment of Abbe-Porter. The theoretical calculations and explanations are given in most text books on diffractive optics'; see for instance the book of Goodman ${ }^{1}$. We used in object plane $\Pi_{0}$ a square grating of spatial period $\delta_{0}=50 \mu \mathrm{m}$ which was in fact nothing else than a negative photo of a grid. In the Fourier plane $\Pi_{F}$ we put a vertical slit, the width of which is given by formula 7 . Alternatively it is possible to use, as vertical slit, the negative photo of a line of width $\delta_{\mathrm{F}}$. That slit has to be well positioned along the line between the light source and the eye. The position of the other components is not critical. When the slit is wide open, there is no filter action, 
and the eye sees a square grid on its retina. When the slit width is reduced to $\delta_{F}$, then the eye sees only horizontal lines, demonstrating the filtering action of the vertical slit (figure 7). When the slit is turned horizontally, only vertical lines are seen in the eye. A special effect occurs when the slit is rotated over $45^{\circ}$ (figure 8). Then only straight lines forming an angle of $-45^{\circ}$ are seen in the eye, although those lines are not present in the original object! Iti is as if the slit creates new lines which where not present in the original object. This looks very strange because the filter is only a passive filter which only absorbs some of the spatial frequencies!

\section{OTHER 4-f EXPERIMENTS}

Once the basic setup is understood, other experiments can be demonstrated. They are illustrated in the next figures. Figure 9 shows an object consisting of two interwoven letters, one with a vertical and the other with a horizontal gratings superposed on it. Because of this grating, both letters have a very distinct spectrum. They can be separated by using a horizontal (resp. vertical) slit. 


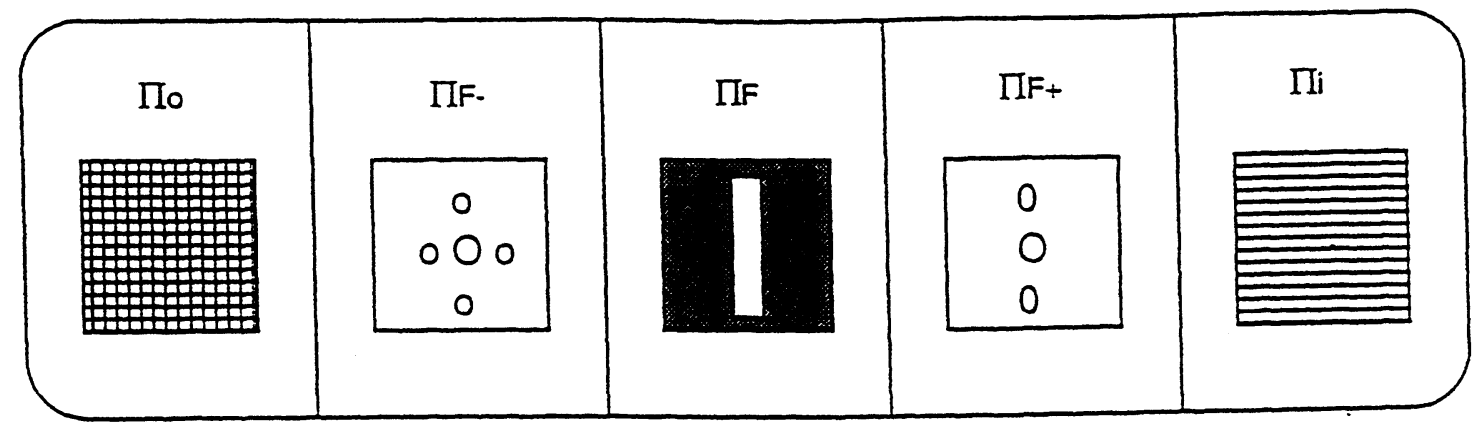

Figure 7 : Different transverse planes in setup of figure 6

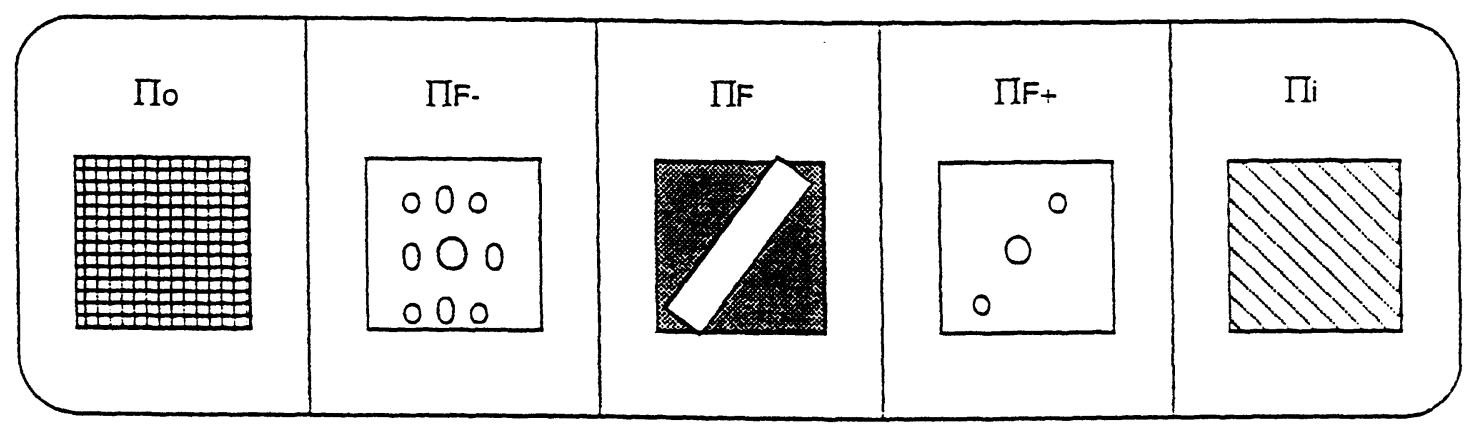

Figure 8 : creatury skew lines with a square grid

Figure 9

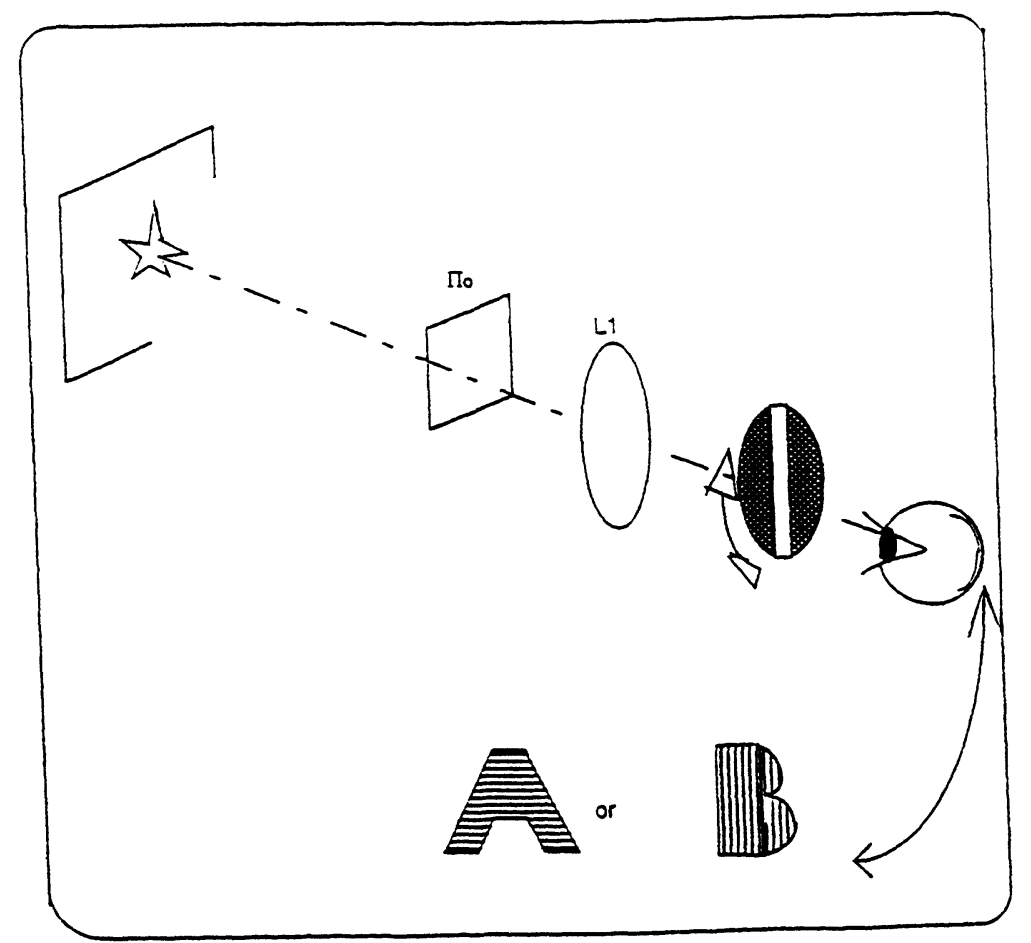




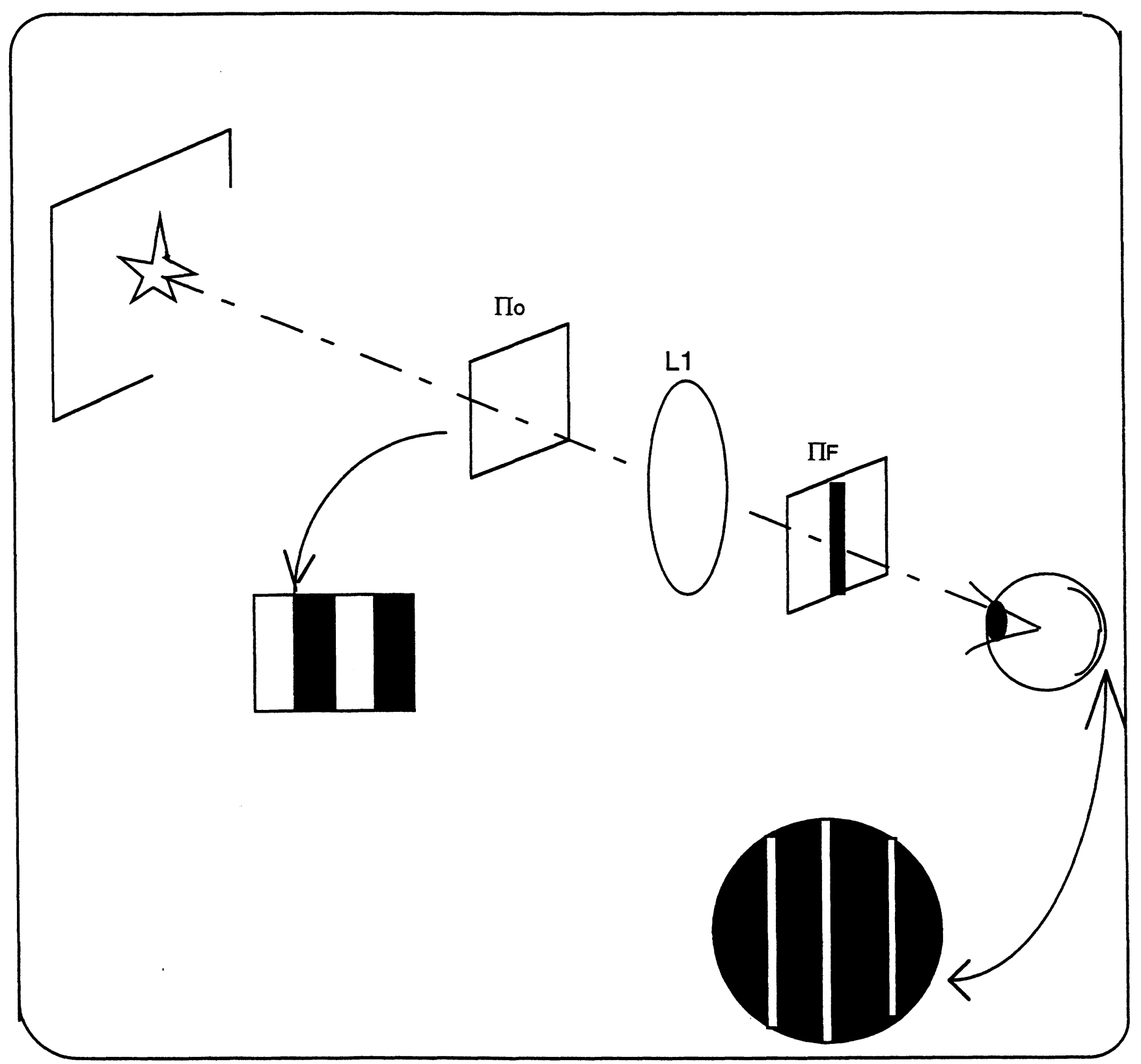

Fig. 10: edge enhancement by absorption of low spatial frequencies 


\section{REFERENCES}

1. Josepth W. Goodman, Introduction to Fourier optics, McGraw-Hill, 1996.

2. D. Sliney and M. Wolbarsht, Safety with lasers and other optical sources, Plenum Press, 1980.

3. J.P. Provost and J.L. Feménias, Optique, vol. 4 : exercices et problèmes d'optique physique, CEDIC/Fernand Nathan Edition, Paris, 1982.

4.Catalog EALING, 1969. 